ISSN 0103-5150

Fisioter. Mov., Curitiba, v. 23, n. 3, p. 429-437, jul./set. 2010

Licenciado sob uma Licença Creative Commons

\title{
Efeitos do exercício resistido de membros superiores na força muscular periférica e na capacidade funcional do paciente com DPOC
}

\author{
Effects of the resistance exercise in upper limb on peripheral muscular \\ strength and functionality of COPD patient
}

\section{Daniela Ike ${ }^{[a]}$, Mauricio Jamami ${ }^{[\mathrm{b}]}$, Diego Marmorato Marino ${ }^{[\mathrm{c}]}$, Gualberto Ruas ${ }^{[\mathrm{d}]}$, Bruna Varanda Pessoa ${ }^{[\mathrm{e}]}$, Valéria Amorim Pires Di Lorenzo ${ }^{[\mathrm{f}]}$}

[a] Mestranda do Programa de Pós-Graduação em Fisioterapia na Universidade Federal de São Carlos (UFSCar), São Carlos, SP - Brasil, e-mail: daniike@yahoo.com.br

[b] Professor Doutor do Departamento de Fisioterapia da Universidade Federal de São Carlos (UFSCar), São Carlos, SP - Brasil, e-mail: jamami@ufscar.br

[c] Mestrando do Programa de Pós-Graduação em Fisioterapia na Universidade Federal de São Carlos (UFSCar), São Carlos, SP - Brasil, e-mail: diego.marmorato@itelefonica.com.br

[d] Mestrandos do Programa de Pós-Graduação em Fisioterapia na Universidade Federal de São Carlos (UFSCar), São Carlos, SP - Brasil, e-mail: gualbertoruas@yahoo.com.br

[e] Doutoranda do Programa de Pós-Graduação em Fisioterapia na Universidade Federal de São Carlos (UFSCar), São Carlos, SP - Brasil, e-mail: bruna.varanda@itelefonica.com.br

[f Professora Doutora do Departamento de Fisioterapia da Universidade Federal de São Carlos (UFSCar), São Carlos, SP - Brasil, e-mail: vallorenzo@ufscar.br

\section{Resumo}

Introdução: A disfunção muscular periférica na doença pulmonar obstrutiva crônica (DPOC) contribui diretamente para a intolerância ao exercício físico, porém ainda não há consenso sobre estratégias adequadas de treinamento físico para esses pacientes. Objetivo: Avaliar o efeito do exercício resistido de membros superiores (MMSS) em pacientes com DPOC moderada a muito grave no ganho de força e na capacidade funcional. Métodos: Doze pacientes com DPOC foram divididos em dois grupos: controle (GC) e treinado (GT). O GT realizou treinamento de força, três vezes por semana, durante seis semanas, com carga de $80 \%$ de uma repetição máxima (RM). Antes e após o tratamento, foram realizados os testes de $1 \mathrm{RM}$ e o Pegboard and Ring Test (PBRT) em ambos os grupos. Resultados: Após o tratamento, verificou-se aumento significativo da força muscular no GT (aumento de 52\% no 
supino sentado e $22 \%$ no pulley, com p < 0,05); e quanto ao PBRT, não houve diferença significativa em ambos os grupos. Conclusão: O treinamento de força de MMSS com duração de seis semanas foi capaz de aumentar a força muscular, mas não a funcionalidade de pacientes com DPOC moderada a muito grave.

Palavras-chave: Reabilitação. Fisioterapia. Membros superiores. Doença pulmonar obstrutiva crônica.

\begin{abstract}
Introduction: Peripheral muscle dysfunction in the chronic obstructive pulmonary disease (COPD) contributes for the intolerance exercise, but there was no consensus about appropriate strategies of exercise training for these patients. Objective: The aim of this study was to assess the effect of the resistance exercise in upper limb in COPD patients (from moderate to very severe cases) on the muscular strength and functional capacity. Methods: Twelve patients with COPD were separated into two groups: control (GC) and trained (GT). The GT carried out strength training three times a week, during six weeks, starting with load of $80 \%$ of one repetition maximum (1 RM). Before and after the treatment were carried out the 1 RM test and the Pegboard and Ring Test (PBRT) in both groups. Results: After the treatment, there was a significant increase of the strength muscle in the GT (increase of 52\% in the supine and $22 \%$ in the pulley, with $p<0.05)$; and in the PBRT there were no significant difference in both groups. Conclusion: The upper limb strength training during six weeks was able to improve the muscle strength, but not the functionality of COPD patients (from moderate to very severe cases).
\end{abstract}

Keywords: Rehabilitation. Physiotherapy. Upper limb. Chronic obstructive pulmonary disease.

\title{
Introdução
}

A doença pulmonar obstrutiva crônica (DPOC) é caracterizada pela presença de obstrução ou limitação crônica do fluxo aéreo que não é totalmente reversível. A limitação ao fluxo aéreo é usualmente progressiva e está associada a uma resposta inflamatória anormal dos pulmões a partículas ou gases nocivos (1).

Embora a DPOC acometa os pulmões, há diversas manifestações sistêmicas relacionadas a esta enfermidade, que incluem intolerância ao exercício físico (2), disfunção muscular periférica (3), alterações nutricionais (4) e exacerbações recorrentes levando a hospitalizações (5). A perda de massa muscular, alterações na fibra do músculo e no fluxo sanguíneo, além de acidose láctica precoce durante o exercício, também contribuem para a intolerância ao exercício físico (6).

A redução da força muscular é proporcional à redução da massa muscular e, pelo fato dos membros superiores (MMSS) serem usados extensivamente nas atividades de vida diária, essa perda ocorre predominantemente nos membros inferiores (MMII) $(7,8)$. Entretanto, em indivíduos com DPOC grave, atividades diárias simples que exigem o uso dos MMSS são pouco toleradas, pelo fato de estarem associadas a alterações ventilatórias e metabólicas significativas $(9,10)$. A simples elevação dos membros superiores altera o recrutamento muscular ventilatório e postural, resultando em assincronia toracoabdominal, aumento da sensação de dispneia em tempo mais curto (11) e término do exercício em cargas mais baixas (12).

Nesse contexto, vários tratamentos têm sido propostos no sentido de minimizar as disfunções, assim como na tentativa de limitar a progressão da doença. Um componente importante no tratamento da DPOC é a Reabilitação Pulmonar (RP), que atualmente engloba inúmeros recursos e métodos de treinamento físico em geral e muscular respiratório.

O treinamento dos músculos periféricos, especificamente, é considerado um componente essencial de um programa de RP em pacientes com DPOC, mas o recondicionamento físico nesses pacientes tem enfocado o treinamento de endurance, que, por sua vez, não leva a alterações na massa muscular ou força, ao contrário do exercício resistido, que pode reduzir a fraqueza muscular periférica e melhorar a capacidade ao exercício (13). 
Efeitos do exercício resistido de membros superiores na força muscular periférica e na capacidade funcional do paciente com DPOC

Considerando que ainda não há consenso sobre qual a melhor estratégia de treinamento de MMSS em pacientes com DPOC (14), o objetivo deste estudo foi avaliar o efeito do exercício resistido de MMSS no ganho de força muscular periférica e na capacidade funcional em pacientes com DPOC.

\section{Material e método}

\section{Amostra}

Participaram deste estudo 12 indivíduos com diagnóstico clínico de DPOC moderada a muito grave, na faixa etária de 50 a 82 anos ( $69 \pm 8$ anos), sendo nove homens e três mulheres.

Os critérios de inclusão foram: volume expiratório forçado no primeiro segundo $\left(\mathrm{VEF}_{1}\right)<50 \%$ do previsto e razão do volume expiratório forçado no primeiro segundo pela capacidade vital forçada $\left(\mathrm{VEF}_{1} /\right.$ $\mathrm{CVF})<70 \%$, constatado pela espirometria pós-broncodilatador (BD) (15); idade igual ou superior a 50 anos; em condição clínica estável, sem períodos de agudização da doença e sem infecções respiratórias por pelo menos um mês antes da avaliação inicial; sedentários; não fumantes ou ex-fumantes, recebendo tratamento medicamentoso; índice de massa corporal (IMC) $<30 \mathrm{~kg} / \mathrm{m}^{2}$ (16).

Foram excluídos da amostra os indivíduos que apresentaram doenças cardiovasculares, neurológicas e/ou osteoarticulares que impedissem a realização segura dos exercícios apresentados no protocolo experimental; indivíduos que apresentaram saturação periférica de oxigênio $\left(\mathrm{SpO}_{2}\right)$ abaixo de $85 \%$; ou aqueles que não aceitaram participar de programa com reavaliações após seis semanas.

Todos os indivíduos foram esclarecidos e orientados quanto à natureza e ao significado do estudo proposto e assinaram um termo de consentimento livre e esclarecido, aprovado pelo Comitê de Ética da Instituição (protocolo n. 193/2008), declarando autorização para a participação neste estudo, em atendimento à Resolução 196/96 do Conselho Nacional de Saúde (CNS).

\section{Procedimento experimental}

Foram avaliados inicialmente 39 pacientes, mas uma grande parte da amostra não apresentou as características solicitadas nos critérios de inclusão. Destes pacientes, 16 foram selecionados para participar do estudo, no entanto, três desistiram por problemas pessoais e um abandonou o estudo. Dessa forma, 12 pacientes concluíram o tratamento e foram divididos aleatoriamente em dois grupos: Controle $(\mathrm{GC} ; \mathrm{n}=7)$ e Treinado (GT; $\mathrm{n}=5)$.

Todos os indivíduos realizaram um teste espirométrico pré e pós-broncodilatador (BD) no aparelho Spirobank $G^{\circledR}$ (MIR, RS232), sob a responsabilidade de um pneumologista e segundo as normas da American Thoracic Society (17), sendo obtidas as medidas de $\mathrm{VEF}_{1}, \mathrm{CVF}$, e relação $\mathrm{VEF}_{1} / \mathrm{CVF}$. Os valores obtidos foram comparados com os previstos segundo Knudson et al. (18).

Inicialmente foi realizada uma avaliação constituída de anamnese e exame físico, e os indivíduos foram orientados a informar sobre qualquer mudança na medicação durante o período do estudo.

Após esta avaliação, foram aplicados os seguintes testes de MMSS: o teste de uma Repetição Máxima $(1 \mathrm{RM})$ e o Pegboard and Ring Test (PBRT), feitos em dias alternados para evitar a fadiga muscular.

\section{Determinação de uma Repetição Máxima (1 RM)}

A determinação de uma repetição máxima (1 RM) consiste na capacidade do deslocamento do maior peso por toda a extensão do movimento articular (19). Este método foi utilizado tanto para determinar a carga a ser utilizada no treinamento de força de MMSS, quanto para avaliar a força muscular pré e pós-tratamento. Destaca-se ainda que no primeiro dia da avaliação foi realizada uma adaptação do voluntário ao equipamento, 
que constou de uma estação de musculação (KENKORP ${ }^{\circledR}$ EMK2710), envolvendo os seguintes exercícios e músculos: supino sentado (peitoral maior, deltoide e tríceps) e pulley superior frontal (grande dorsal, redondo maior, deltoide posterior, romboide, trapézio médio e inferior e bíceps braquial).

O teste para a determinação de $1 \mathrm{RM}$ foi realizado da seguinte maneira: um breve aquecimento (cinco minutos) com exercícios ativo-livres de MMSS (diagonal primitiva de Kabat e flexo-extensão de ombro), seguido de um protocolo crescente, ou seja, as cargas foram aumentadas progressivamente até a obtenção da maior carga deslocada na amplitude articular total (20). Este procedimento foi realizado para os dois movimentos citados anteriormente, sendo que a ordem foi escolhida por sorteio para o primeiro paciente, sendo seguido para todos os pacientes posteriormente. Ressalta-se que houve um intervalo de cinco minutos entre cada tentativa para monitorizar a saturação periférica de oxigênio $\left(\mathrm{SpO}_{2}\right)$ e a frequência cardíaca (FC), por meio de um oxímetro de pulso $\left(\right.$ Nonin $^{\circledR}$, modelo $\left.8500 \mathrm{~A}\right)$; a pressão arterial (PA), por meio de um esfigmomanômetro aneroide (Dyasist ${ }^{\circledR}$ ); e a dispneia e o cansaço de MMSS pela Escala de Borg CR-10 modificada. Foram realizadas até cinco tentativas para se determinar a 1 RM; caso ultrapassasse essas tentativas, outro dia de avaliação era agendado para realizar novo teste.

Durante o esforço físico, os indivíduos foram orientados a não realizar a manobra de Valsalva, e foi ensinada a técnica da respiração com freno-labial.

\section{Pegboard and Ring Test (PBRT)}

O Pegboard and Ring Test é um teste de MMSS sem suporte, desenvolvido por Celli et al. (11) e validado por Zhan et al. (21) para avaliar a capacidade funcional dos MMSS em indivíduos com DPOC.

Para esse teste, os indivíduos permaneceram sentados em frente a um quadro, contendo dois pinos posicionados na altura de seu ombro e outros dois pinos colocados $20 \mathrm{~cm}$ acima do ombro. Dez argolas foram colocadas em cada um dos dois pinos inferiores.

Os indivíduos foram instruídos a usar ambas as mãos e mover uma argola de cada vez, iniciando com o lado dominante do indivíduo, deslocando-as do pino inferior para o superior. Após posicionarem todas as argolas do nível inferior para o superior, os indivíduos voltaram a posicionar as argolas para o nível inferior e assim sucessivamente (22).

A pontuação total do teste foi a quantidade de argolas deslocadas em seis minutos, sendo permitido ao indivíduo parar para descansar por motivo de fadiga, dispneia ou outro desconforto e voltar a realizar o teste assim que se sentisse mais confortável, mantendo o cronômetro acionado para medição do tempo (22).

\section{Programa de tratamento}

Tanto os indivíduos do GC quanto os do GT foram submetidos a um tratamento que constou de três sessões semanais, com duração de 40 minutos cada, durante seis semanas consecutivas. O GC realizou sessões constituídas de condutas de higiene brônquica e reeducação funcional respiratória.

As sessões do GT iniciaram com aquecimento (cinco minutos) seguido de três séries de oito repetições de cada exercício (supino sentado e pulley superior frontal), com intervalo de dois minutos entre as séries e cinco minutos de descanso entre cada exercício; e ao fim, foram realizados mais cinco minutos de alongamento dos MMSS. A carga foi reajustada de acordo com os valores obtidos no teste de $1 \mathrm{RM}$, realizado a cada seis sessões, mantendo-se o percentual de $80 \%$ de 1 RM.

\section{Análise estatística}

Os dados foram analisados pelo software Statistical Package for Social Sciences for Windows versão 13.0 (SPSS $^{\circledR}$ Inc, Chicago, IL). 
Efeitos do exercício resistido de membros superiores na força muscular periférica e na capacidade funcional do paciente com DPOC

A distribuição dos dados foi verificada pelo teste de normalidade de Shapiro-Wilk. Os dados foram expressos em médias e desvios padrão. Para comparação dos dados pré e pós-tratamento foi utilizado o teste $t$ de Student, com significância de p $<0,05$.

\section{Resultados}

As características demográficas, antropométricas e espirométricas dos indivíduos com DPOC de cada grupo, obtidas antes do tratamento, são apresentadas na Tabela $1 . \mathrm{Na}$ análise intergrupos não se constatou diferença significante, atestando assim a homogeneidade da amostra.

Tabela 1 - Características demográficas, antropométricas e espirométricas dos indivíduos com DPOC dos grupos controle (GC) e treinado (GT)

\begin{tabular}{lcc}
\hline & GC $(\mathbf{n}=7)$ & GT $(\mathbf{n}=\mathbf{5})$ \\
\hline Idade (anos) & $70,4 \pm 8,5$ & $67,8 \pm 7,4$ \\
Sexo, H/M & $5 / 2$ & $4 / 1$ \\
Altura (m) & $1,60 \pm 0,1$ & $1,60 \pm 0,1$ \\
Peso $(\mathrm{kg})$ & $67,8 \pm 5,8$ & $64,3 \pm 11,8$ \\
$\mathrm{IMC}\left(\mathrm{kg} / \mathrm{m}^{2}\right)$ & $25,7 \pm 3,4$ & $23,5 \pm 2,5$ \\
$\operatorname{VEF}_{1}(\%$ prev $)$ & $34,3 \pm 8,1$ & $30,9 \pm 18,9$ \\
$\operatorname{VEF}_{1} / \mathrm{CVF}(\%)$ & $49,6 \pm 14,3$ & $42,7 \pm 26,1$ \\
\hline
\end{tabular}

Legenda: IMC: Índice de massa corporal; $\mathrm{VEF}_{1}$ : Volume expiratório forçado no primeiro segundo; $\mathrm{VEF}_{1} / \mathrm{CVF}$ : Razão do volume expiratório forçado no primeiro segundo pela capacidade vital forçada. Os valores correspondem à média \pm DP.

\section{Força muscular dos MMSS}

Após seis semanas de tratamento, verificou-se aumento significativo da força muscular somente no GT, sendo de $52 \%$ no exercício de supino $(p=0,0008)$ e de $22 \%$ no exercício de pulley $(p=0,03)$. Destaca-se que no GC não houve diferença significativa $(p>0,05)$ da força muscular nos períodos pré e pós-tratamento (Tabela 2).

Tabela 2 - Carga deslocada no teste de 1 Repetição Máxima (1RM) nos exercícios de supino e pulley dos grupos Controle (GC) e Treinado (GT) nas fases pré e pós-tratamento

\begin{tabular}{lccccc}
\hline & \multicolumn{3}{c}{ GC $(\mathrm{n}=7)$} & & \multicolumn{2}{c}{ GT $(\mathrm{n}=5)$} \\
\cline { 2 - 3 } \cline { 5 - 6 } & Pré & Pós & & Pré & Pós \\
\hline Supino $(\mathrm{kg})$ & $39 \pm 11,6$ & $48 \pm 11,9$ & & $48 \pm 14$ & $73 \pm 17,6^{*}$ \\
Pulley $(\mathrm{kg})$ & $25 \pm 6,9$ & $26 \pm 5,2$ & & $27 \pm 7,6$ & $33 \pm 7,9^{*}$ \\
\hline
\end{tabular}

Nota: Os valores correspondem à média \pm DP. Análise Intragrupo: * diferença estatística $(\mathrm{p}<0,05)$. 


\section{Pegboard and Ring Test (PBRT)}

Em relação ao desempenho no PBRT, constatou-se que tanto no GC como no GT, não se observou diferença significativa $(\mathrm{p}>0,05)$ entre o número de argolas movidas antes e após as seis semanas de tratamento (Tabela 3).

Tabela 3 - Número de argolas movidas no Pegboard and Ring Test (PBRT) nas fases pré e pós-tratamento nos grupos Controle (GC) e Treinado (GT)

\begin{tabular}{ccccccc}
\hline & \multicolumn{3}{c}{ GC $(\mathrm{n}=7)$} & & \multicolumn{2}{c}{ GT $(\mathrm{n}=5)$} \\
\cline { 2 - 3 } \cline { 5 - 6 } & Pré & Pós & & Pré & Pós \\
\hline Argolas movidas $\left(\mathrm{n}^{\circ}\right)$ & $193 \pm 44$ & $199 \pm 63(\mathrm{~ns})$ & & $189 \pm 35$ & $199 \pm 21(\mathrm{~ns})$ \\
\hline
\end{tabular}

Nota: Os valores correspondem à média \pm DP; $n^{\circ}$ : repetições no PBRT; ns: não apresentou diferença significativa.

\section{Discussão}

O objetivo deste estudo foi avaliar o efeito do exercício resistido de MMSS no ganho de força e na capacidade funcional em pacientes com DPOC moderada a muito grave. Os resultados apontaram que, embora a capacidade funcional não tenha apresentado diferença significativa em ambos os grupos, o treinamento de força de MMSS mostrou-se importante na reabilitação do paciente com DPOC moderada a muito grave, porque, mesmo treinando com alta intensidade ( $80 \%$ de $1 \mathrm{RM})$, foi bem tolerado e resultou em aumento da força muscular.

Um crescente número de evidências científicas tem confirmado a presença de disfunção muscular em pacientes com DPOC. Entre elas destacam-se: a redução das fibras tipo I; a atrofia de fibras tipo I e II; a capilaridade reduzida; e níveis alterados de enzimas metabólicas (13). Allard et al. (23) verificaram que a força isométrica dos músculos quadríceps, do handgripe dos músculos respiratórios de pacientes com DPOC estava reduzida, assim como a capacidade ventilatória desses indivíduos, contribuindo independentemente para a redução da capacidade ao exercício físico.

Segundo Simpson et al. (24), a piora mais evidente na capacidade ao exercício foi encontrada em indivíduos com obstrução muito grave, além da maior redução nos índices de força muscular esquelética. Nesse mesmo estudo houve o aumento de 33\% de 1 RM do exercício de membro superior, comparando pré e pós-treinamento. Ortega et al. (25) também realizaram treinamento de força de MMSS e MMII em indivíduos com DPOC com obstrução moderada a grave, e constataram um aumento da força muscular em todos os exercícios realizados após o tratamento. Nosso estudo corrobora com os resultados desses autores, uma vez que o treinamento de força durante seis semanas promoveu aumento da força muscular nos exercícios de supino e pulley ( $52 \%$ e $22 \%$, respectivamente), avaliado pelo teste de 1 RM.

Cabe ressaltar que, no nosso estudo, mesmo sendo um período de treinamento menor do que aqueles já mencionados $(24,25)$, o aumento de força foi significativo para o grupo treinado. Hickson et al. (26) e O’Bryant et al. (27) mostraram que em períodos de 11 a 16 semanas (treinamento de curta duração), o maior aumento de força ocorre entre as primeiras quatro a oito semanas.

Além do tempo de treinamento, outro fator importante é a intensidade (carga). Casaburi et al. (28) mostraram melhoras significativas nas respostas de exercícios com carga máxima e submáxima em treinamento de alta intensidade $(70-80 \%$ da carga máxima). Em estudo recente, Hoff et al. (29) realizaram treinamento de MMII em pacientes com DPOC com carga de 90\% de 1 RM e notaram melhora significativa da força muscular periférica (aumento de 105\%). Esses dados indicam que o treinamento 
de força de alta intensidade resulta em maiores adaptações fisiológicas (29), além de ser seguro e bem tolerado (24).

Com relação ao PBRT, os resultados mostraram que não houve diferença significativa no número de argolas movidas no GC e GT. Isso pode ser explicado principalmente pelo número reduzido de pacientes em cada grupo, além do fato desse teste avaliar a capacidade funcional, a qual não foi o objetivo desse estudo em termos de treinamento.

Apesar de ter sido desenvolvido há aproximadamente 20 anos por Celli, Rassulo e Make (11), o PBRT tem sido pouco utilizado na avaliação dos MMSS em indivíduos com DPOC (30, 12). Porém, por se tratar de um teste validado, simples e de baixo custo para avaliar a capacidade funcional dos MMSS, apresenta um grande potencial clínico e científico. Considerando que a sensação de dispnea em atividades de MMSS é um dos maiores problemas de pacientes com DPOC moderada a grave (22), o PBRT pode se tornar uma importante medida da eficácia da RP. Apesar de não constatarmos diferença significativa no número de argolas deslocadas após o tratamento, ambos os grupos mantiveram-se estáveis.

\section{Considerações finais}

A partir dos resultados obtidos neste estudo, pode-se concluir que o treinamento de força de MMSS com duração de seis semanas não resultou em melhora na funcionalidade, mas foi capaz de aumentar a força muscular periférica em pacientes com DPOC moderada a muito grave, sendo uma alternativa a ser incluídas nos programas de RP.

A implicação clínica deste estudo está no fato de que o treinamento de força para pacientes com obstrução grave do fluxo aéreo pode ser uma alternativa mais adequada, se comparada ao treinamento de endurance, pela possibilidade de se trabalhar com cargas mais altas com menor sensação de dispneia. Todavia, ainda são necessários mais estudos envolvendo treinamento de força isolado e com amostras mais consistentes.

\section{Referências}

1. Pauwels RA, Buist AS, Ma P, Jenkins CR, Hurd SS. Global strategy for the diagnosis, management, and prevention of chronic obstructive pulmonary disease GOLD: executive summary. Respir Care. 2001;46(8):798-825.

2. Gosselink R, Troosters T, Decramer M. Peripheral muscle weakness contributes to exercise limitation in COPD. Am J Respir Crit Care Med. 1996;153(3):976-80.

3. Mador MJ, Denis O, Aggarwal A, Kufel TJ. Quadriceps fatigability after single muscle exercise in patients with chronic obstructive pulmonary disease. Am J Respir Crit Care Med. 2003;168(1):102-8.

4. Schols AM, Soeters P, Dingermans M, Mostert R, Frantzen PJ, Wouters EF. Prevalence and characteristics of nutritional depletion in patients with stable COPD eligible for pulmonary rehabilitation. Am Rev Respir Dis. 1993;147(5):1151-6.

5. Connors AF Jr, Dawson NV, Thomas C, Harrel FE Jr, Desbiens N, Fulkerson WJ, et al. Outcomes following acute exacerbation of severe chronic obstructive pulmonary disease: the SUPPORT Investigators (Study to Understand Prognosis and Preferences for Outcomes and Risks of Treatments). Am J Respir Crit Care Med. 1996;154(4 Pt 1): 959-67.

6. Nici L, Donner C, Wouters E, Zuwallack R, Ambrosino N, Bourbeau J. American Thoracic Society/European Thoracic Society Pulmonary Rehabilitation Writing Committee. Skeletal muscle dysfunction in chronic obstructive pulmonary disease. Am J Respir Crit Care Med. 2006;173(12):1390-413.

7. Bernard S, Leblanc P, Whittom F, Carrier G, Jobin J, Belleau R, et al. Peripheral muscle weakness in patients with chronic obstructive pulmonary disease. Am J Respir Crit Care Med. 1998;158:629-34. 
8. Dourado VZ, Tanni SE, Vale SA, Faganello MM, Sanchez FF, Godoy I. Manifestações sistêmicas na doença pulmonar obstrutiva crônica. J Bras Pneumol. 2006;32(2):161-71.

9. Velloso M, Stella SG, Cendon S, Silva AC, Jardim JR. Metabolic and ventilatory parameters of four activities of daily living accomplished with arms in COPD patients. Chest. 2003;123(4):1047-53.

10. Jeng C, Chang W, Wai PM, Chou CL. Comparison of oxygen consumption in performing daily activities between patients with chronic obstructive pulmonary disease and a healthy population. Heart Lung. 2003; 32(2):121-30.

11. Celli BR, Rassulo J, Make BJ. Dyssynchronous breathing during arm not leg exercise in patients with chronic airflow obstruction. N Engl J Med. 1986;314(23):1485-90.

12. Criner GJ, Celli BR. Effect of unsupported arm exercise on ventilatory recruitment in patients with severe chronic airflow obstruction. Am Rev Respir Dis. 1988;138(4):856-61.

13. O'Shea SD, Taylor NF, Paratz J. Peripheral muscle strength training in COPD: a systematic review. Chest. 2004;126(3):903-14.

14. Celli BR. Pulmonary rehabilitation in patients with COPD. Am J Respir Crit Care Med. 1995;152(3): $861-4$.

15. Rabe KF, Hurd S, Anzueto A, Barnes PJ, Buist SA, Calverley P. Global strategy for the diagnosis, management, and prevention of chronic obstructive pulmonary disease: GOLD executive summary. Am J Respir Crit Care Med. 2007;176(6):532-55.

16. Coutinho W. Consenso latino-americano em obesidade. [acesso em 12 set. 2008]. Disponível em: www.abeso.org. $\mathrm{br} /$ consenso.doc

17. American Thoracic Society. Standardization of spirometry. Eur Respir. J 2005;26(2):319-38.

18. Knudson RJ, Slatin RC, Lebowitz MD, Burrows B. The maximal expiratory flow-volume curve: normal standards, variability and effects of age. Am Rev Respir Dis. 1976;113(5):587-600.

19. Powers SK, Howley ET. Testes de esforço para a avaliação do desempenho. In: Powers SK, Howley ET. Fisiologia do exercício: teoria e aplicação ao condicionamento e ao desempenho. São Paulo: Manole; 2000. p. 371-90.

20. Neder JR, Nery LE. Determinação de 1 repetição máxima. In: Neder JR, Nery LE. Fisiologia clínica do exercício: teoria e prática. São Paulo: Artes Médicas; 2003. p. 178-179.

21. Zhan S, Cerny FJ, Gibbons WJ, Mador MJ, Wu Y-W. Development of an unsupported arm exercise test in patients with chronic obstructive pulmonary disease. J Cardiopulm Rehabil. 2006;26(3):180-7.

22. Vilaró J, Resqueti VR, Fregonezi GAF. Avaliação clínica da capacidade do exercício em pacientes com doença pulmonar obstrutiva crônica. Rev Bras Fisioter. 2008;12(4):249-59.

23. Allard C, Jones NL, Killian KJ. Static peripheral skeletal muscle strength and exercise capacity in patients with chronic airflow limitation. Am Rev Respir Dis. 1990;139:A90.

24. Simpson K, Killian K, McCartney N, Stubbing DG, Jones NL. Randomized controlled trial of weightlifting exercise in patients with chronic airflow limitation. Thorax. 1992;47(2):70-5.

25. Ortega F, Toral J, Cejudo P, Villagomez R, Sanchez H, Castillo J, et al. Comparison of effects of strength and endurance training in patients with chronic obstructive pulmonary disease. Am J Respir Crit Care Med. 2002; 166(5):669-74.

26. Hickson RC, Hidaka K, Foster C. Skeletal muscle fiber type, resistance training, and strength-related performance. Med Sci Sports Exerc. 1994;26(5):593-8. 
27. O’Bryant HS, Byrd R, Stone MH. Cycle ergometer performance and maximum leg and hip strength adaptations to two different methods of weight-training. J Appl Sport Sci Res. 1988;2(2):27-30.

28. Casaburi R, Patessio A, Ioli F, Zanaboni S, Donner CF, Wasserman K. Reductions in exercise lactic acidosis and ventilation as a result of exercise training in patients with obstructive lung disease. Am Rev Respir Dis. 1991;143(1):9-18.

29. Hoff J, Tjonna AE, Steinshmn S, Hoydal M, Richardson RS, Hegerud J. Maximal strength training of the legs in COPD: a therapy for mechanical inefficiency. Med Sci Sports Exerc. 2007;39(2):220-6.

30. Bauldoff GS, Hoffman LA, Sciurba F, Zullo TG. Home-based, upper-arm exercise training for patients with chronic obstructive pulmonary disease. Heart Lung. 1996;25(4):288-94.

Recebido: 26/01/2009

Received: 01/26/2009

Aprovado: 07/05/2010

Approved: 05/07/2010

Revisado: 24/06/2010

Reviewed: 06/24/2010 